\title{
Exact nonparaxial beams of the scalar Helmholtz
}

\section{equation}

\author{
G. Rodríguez-Morales, S. Chávez-Cerda \\ Instituto Nacinal de Astrofísica, Optica y Electrónica Apdo Postal 51/216, Puebla, \\ Pue. 72000, México
}

\begin{abstract}
It is shown that three-dimensional nonparaxial beams are described by the oblate spheroidal exact solutions of the Helmholtz equation. For the first time, their beam behaviour is investigated and their corresponding parameters are defined. Using the fact that the beam width of the family of paraxial Gaussian beams is described by an hyperbola, the connection between the physical parameters of nonparaxial spheroidal beam solutions and those of paraxial beams is formally stablished. These results are also helpful to investigate the exact vector nonparaxial beams. (C) 2018 Optical Society of America
\end{abstract} OCIS codes: $350.5500,260.1960,260.2110,140.3410,000.3860$ 
Recent developments in science and technology brings the necessity of revisiting the theory and concepts of nonparaxiality of optical beams. Its understanding has great relevance in the description of optical fields which are tightly focused or, optical beams whose diameter can be of the order of a few wavelengths as can be the case in optical nanolasers.

When studying beams beyond the paraxial approximation, the standard approach, introduced by Lax, has been to start with the paraxial solution and then to include some corrections. $\frac{12344}{2 i g u r o u s l y ~ s p e a k i n g, ~ n o n p a r a x i a l ~ b e a m s ~ a r e ~ s o l u-~}$ tions of the wave equation without the paraxial approximation, in other words, they are solutions of the Helmholtz wave equation $\nabla^{2} E+k^{2} E=0$, with $k$ the wave number. Thus, such kind of solutions must be investigated in order to describe nonparaxial beams.

A class of nonparaxial solutions has been introduced assuming a point source located at a complex position along the $z$-axis. The spherical wave field from this virtual complex source is converted into a directional wave field which remains a rigurous solution of the Helmholtz equation $[5$ This virtual source mode has wavefronts that are approximately oblate spheroidal ${ }^{6}$ This fact has also been discussed by several authors and even have expressed the virtual source mode wave in spheroidal coordinates $\frac{78[910}{10}$ Whatever the coordinate system used, the field expression reduces, under the paraxial approximation, to Gaussian beam! 67891011

The virtual complex source point solutions carry an inherent singularity which makes them inadequate to describe propagating fields near the origin or focal source point ${ }^{68}$ To elliminate this problem a non-singular superposition of incoming and 
outgoing spherical waves has been used ${ }^{68}$ however even this non-singular solution has problems since, to realise them physically, infinite energy is required $\frac{[12}{12}$

In this letter we investigate the solutions of the scalar Helmholtz equation in oblate spheroidal coordinates and we show that they can have a beam behaviour. By identifying that the evolution of the beam width of a Gaussian mode follows an hyperbola we relate the physical parameters of nonparaxial spheroidal beam solutions and those of paraxial beams. This also allows to define quantitatively a threshold between nonparaxial and paraxial beams. In the paraxial limit the oblate spheroidal solution tend to Laguerre-Gaussian beams.

Pure plane, spherical or cylindrical waves of the Helmholz equation, cannot be used, in a strict sense, to describe optical beams if we understand as such those field distributions that are concentrated around an imaginary line that will be identified as the beam propagation axis. Here we show that solutions of the Helmholtz equation in spheroidal coordinates can satisfy this requirement depending on the value of the ellipsoidal parameter (to be defined below).

The propagation axis will be the $z$-axis that will also be the azimuthal axis of the oblate spheroid coordinate system. This coordinates are defined by $x=d\left(\xi^{2}+1\right)^{1 / 2}(1-$ $\left.\eta^{2}\right)^{1 / 2} \cos \phi, y=d\left(\xi^{2}+1\right)^{1 / 2}\left(1-\eta^{2}\right)^{1 / 2} \sin \phi$ and $z=d \xi \eta$, where $d>0$ is the distance from the origin to the foci ${ }^{13144}$ The variation range of the coordinates is determined by the physical problem. In the present case they are $0 \leq \eta \leq 1,-\infty \leq \xi \leq \infty$ and $0 \leq \phi \leq 2 \pi$. The first two coordinates can be related to an eccentric radial coordinate $\rho=\sinh ^{-1} \xi$ and an eccentric angular coordinate by $\theta=\cos ^{-1} \eta$. From the above coordinate transformations, the condition $|\eta|=1$ defines the $z$-axis. 
In oblate spheroidal coordinates $(\xi, \eta, \phi)$ the Helmholtz wave equation $\nabla^{2} E+$ $k^{2} E=0$, is separated into the next set of equations $\frac{1314}{114}$

$$
\begin{gathered}
\frac{d^{2} \Phi}{d \phi^{2}}+m^{2} \Phi=0 \\
\left(\xi^{2}+1\right) \frac{d^{2} R}{d \xi^{2}}+2 \xi \frac{d R}{d \xi}-\left(a_{m n}-c^{2} \xi^{2}+\frac{m^{2}}{\xi^{2}+1}\right) R=0 \\
\left(1-\eta^{2}\right) \frac{d^{2} S}{d \eta^{2}}-2 \eta \frac{d S}{d \eta}+\left(a_{m n}-c^{2} \eta^{2}-\frac{m^{2}}{1-\eta^{2}}\right) S=0
\end{gathered}
$$

where $c=k d$ with $a_{m n}$ is a constant parameter. The spheroidal parameter $c$ is a meassure of how far is the spheroid from a sphere for which $d=0$ and in consequence $c=0$. From Eq. (II) the solutions of Helmholtz equation have the form of a Lamé product $\frac{13}{13}$

$$
E_{n m}(\xi, \eta, \phi)=R_{m n}^{(p)}(\xi ; c) S_{m n}(\eta ; c) e^{i m \phi}
$$

where in the spheroidal radial functions $R_{m n}^{(p)}(\xi ; c)$, the superindex $p$ defines the kind of solution (first kind $p=1$, second kind $p=2$ and so on). With $p=3,4$ the solutions are linear combinations of the first two, namely $R_{m n}^{(3,4)}=R_{m n}^{(1)} \pm i R_{m n}^{(2)}$, and these are what we will use as they, can be used to describe travelling waves. There are two spheroidal angular solutions $S_{m n}^{(1,2)}(\eta ; c)$ but, we exclude the second one since it tends to infinity as it approaches the $z$-axis then, only that of first kind can describe beams of finite energy and so we drop the superindex for this solution.

In order to understand the physics of Eq. (22) we refer to Fig. 1 where the intensity of the modes with $m=n=0$ and $m=n=1$ are shown. One immediately realises that for $c \neq 0$, the wavefield distribution is concentrated around the $z$-axis, in other words, it behaves like a beam. The angular part of (21) determines the transverse pattern of the wavefield while the radial part defines the propagation features. The azimuthal and radial parts of the solution determine the form of the propagating 
phase front, when $m=0$ they are oblate spheroidal, Fig $1 \mathrm{a}$ ), and when $m \neq 0$ the solution describes wavefields with rotating wavefronts, Fig 1b).

To relate spheroidal solutions, Eq. (2) with those of the paraxial wave equation analyse the geometry of paraxial beams. Their beam width $w(z)$ is given by $w^{2}(z)=$ $w_{0}^{2}\left(1+z^{2} / R_{0}^{2}\right)$ where $w_{0}$ is the beam waist and $R_{0}$ is the Rayleigh distance. ${ }^{[15}$ Rewriting this expression we get the hyperbola equation $w^{2}(z) / w_{0}^{2}-z^{2} / R_{0}^{2}=1$ to which we can associate an oblate spheroidal coordinate system, this is shown in Fig. 2. In it, $d$ is the distance to one of the foci and $w_{0}$ is the vertex of the hyperbola. From the geometry of the hyperbola, the equation

$$
R_{0}^{2}=d^{2}-w_{0}^{2}
$$

has to be fulfilled. From the equation of the hyperbola, the angle of the asymptote or far field angle is $\tan \alpha=w_{0} / R_{0}$, this assigns a geometric place to $R_{0}$ in Fig. 2 . We now make the connection between paraxial and spheroidal beams through the common parameter $d$. Sustituting $c=k d$ and $R_{0}=k w_{0}^{2} / 2$ in Eq. (3), after some algebra, we get

$$
w_{0}=\frac{\sqrt{2\left(-1+\sqrt{1+c^{2}}\right)}}{k} .
$$

This equation is the link that connects oblate spheroidal beams and paraxial beams. There must exist a range of values of $c$ for which oblate spheroidal beams describe paraxial beams. This can be obtained, expressing this parameter in terms of the far field angle $c=2 /(\tan \alpha \sin \alpha)$, since paraxial beams cannot diverge at cone angles larger than $\alpha \approx 0.5 \mathrm{rad} \approx \pi / 6^{\frac{15}{5}}$ we have $c_{p} \approx 7$ this value defines the frontier between paraxial and nonparaxial beams. In Fig. 3 we show the plots of the on-axis intensity 
$\left|R_{m n}^{(3,4)}\left(\xi ; c_{p}\right)\right|^{2}$ and transverse profile $S_{m n}\left(\eta ; c_{p}\right)$ of an oblate spheroidal beam in this limit and compare them with the corresponding paraxial beam. Also, for reference, it is plotted the normalised axial intensity for a highly nonparaxial beam with $c<c_{p}$ (inner thin line). The small differences can be attributed to that the paraxial LaguerreGauss beam is in the limit of its validity. In fact, for $c>c_{p}$ the behaviour of the oblate spheroidal beams is even closer to paraxial beams. We have evaluated the wavefield profiles for large values of $c>c_{p}$ and found that the transverse part of the spheroidal beams described by the angular solutions $S_{m n}(\eta ; c)$ tend to the Laguerre-Gauss beam, as expected $\frac{13}{13}$ This similarity occurs earlier for the zero-order mode for $c$ just above $c_{p}$. Finally, notice that from Eq. (4) when $c \rightarrow \infty$ the radial functions $R_{m n}^{(3,4)}$ tend to plane waves for the fundamental order mode and when $c \rightarrow 0$, the nonparaxial oblate spheroidal beams tend to resemble spherical waves.

That the solutions discussed here are different from those obtained with the complex source point method (CSPM) expressed in spheroidal coordinates. ${ }^{78}$ 10 The zero-order nonparaxial beams obtained with the CSPM have a singularity inherited from its construction from a source point ${ }^{12}$ The oblate spheroidal beams presented here do not present any singularity whenever $c \neq 0$. Using the CSPM, $\frac{78910}{10}$ is usually proposed that in the paraxial limit $d$ can take the value of $R_{0}$. From eq. (3) it is clear that this situation can never be possible since it would imply a physical inconsistency, $w_{0}=0$ and a far-field angle $\alpha=0$ (see Fig. 2).

In conlcusion, for the first time, we have demonstrated that spheroidal beams describe paraxial and nonparaxial beam exact solutions of the Helmholtz equation. 
We have established a threshold that separates nonparaxial and paraxial regimes. Higher oblate spheroidal modes reduce in a natural way to Laguerre-Gauss beams when the spheroidal parameter $c$ exceeds the critical value $c_{p}$. The results presented here were in the neighborhood of the paraxial limit and thus the scalar treatment is valid. Our approach extends straightforward to vector nonparaxial beams in Eq. (2),

to obtain the corresponding vectorial components obtained ${ }^{[16]}$ Thorough investigations are underway.

Authors acknowledge to CONACyT. GRM Acknowledge support to CONACyT grant 118631/120329.

\section{References}

1. M. Lax, W. H. Louisell and W. B. McKnight, Phys. Rev. A 11,1365-1370 (1975).

2. S. R. Seshadri, J. Opt. Soc. Am. 19, 2134-2141 (2002) and references therein.

3. A. Ciattoni, B. Crosignani and P. Di Porto, Opt. Commun. 177, 9-13 (2000).

4. Q. Cao and X. Deng, J. Opt. Soc. Am. A 15, 1144-1148 (1998).

5. S. Y. Shin and L. B. Felsen, J. Opt. Soc. Am. 67, 699-700 (1977).

6. C. J. R. Sheppard and S. Saghafi, Phys. Rev. A 57 2971-2979 (1998).

7. B. T. Landesman and H. H. Barrett, J. Opt. Soc. Am. A 5, 1610-1619 (1988).

8. Z. Ulanowski and I. K. Ludlow, Opt. Lett. 25, 1792-1794 (2000).

9. P. Saari, Opt. Express 8, 590-598 (2001).

10. H. G. Kraus, J. Opt. Soc. Am. A 7, 47-65 (1990).

11. G. A. Deschamps, Electron. Lett. 7, 684-685 (1971).

12. J. Lekner, J. Opt. A: Pure Appl. Opt. 3, 407-412 (2001). 
13. C. Flammer, Spheroidal wave functions. (Stanford Univ. Press, Stanford California, 1957).

14. S. Zhang, J. Jin, Computation of special functions. Wiley \& Sons. New York, 1996, chapter 15

15. A. E. Siegman, Lasers, University Science Books, Sausalito CA,1986.

16. J. A. Stratton, Electromagnetic Theory, McGraw-Hill, New York 1941, Chapter VII. 


\section{Figure Captions}

1. Intesity and phase evolution (left), and transverse pattern (right) of oblate spheroidal modes for $c=7$; a) $m=n=0$ b) $m=n=1$.

2. Hyperbolic-elliptic geometry of a paraxial beam defined by the beam width evolution.

3. a) Comparison of on-axis intensity for oblate spheroidal nonparaxial (thick solid line) and paraxial (dashed line) beams. Also it is shown the normalised intensity for a highly nonparaxial beam (thin line). b) Corresponding transverse amplitude profiles at the plane $z=0$. Notice that for the Laguerre paraxial mode $r$ can be obtained from $r^{2}=x^{2}+y^{2}=d^{2}\left(1-\eta^{2}\right)$. 
Fig. 1. 
Fig. 2. 
Fig. 3. 

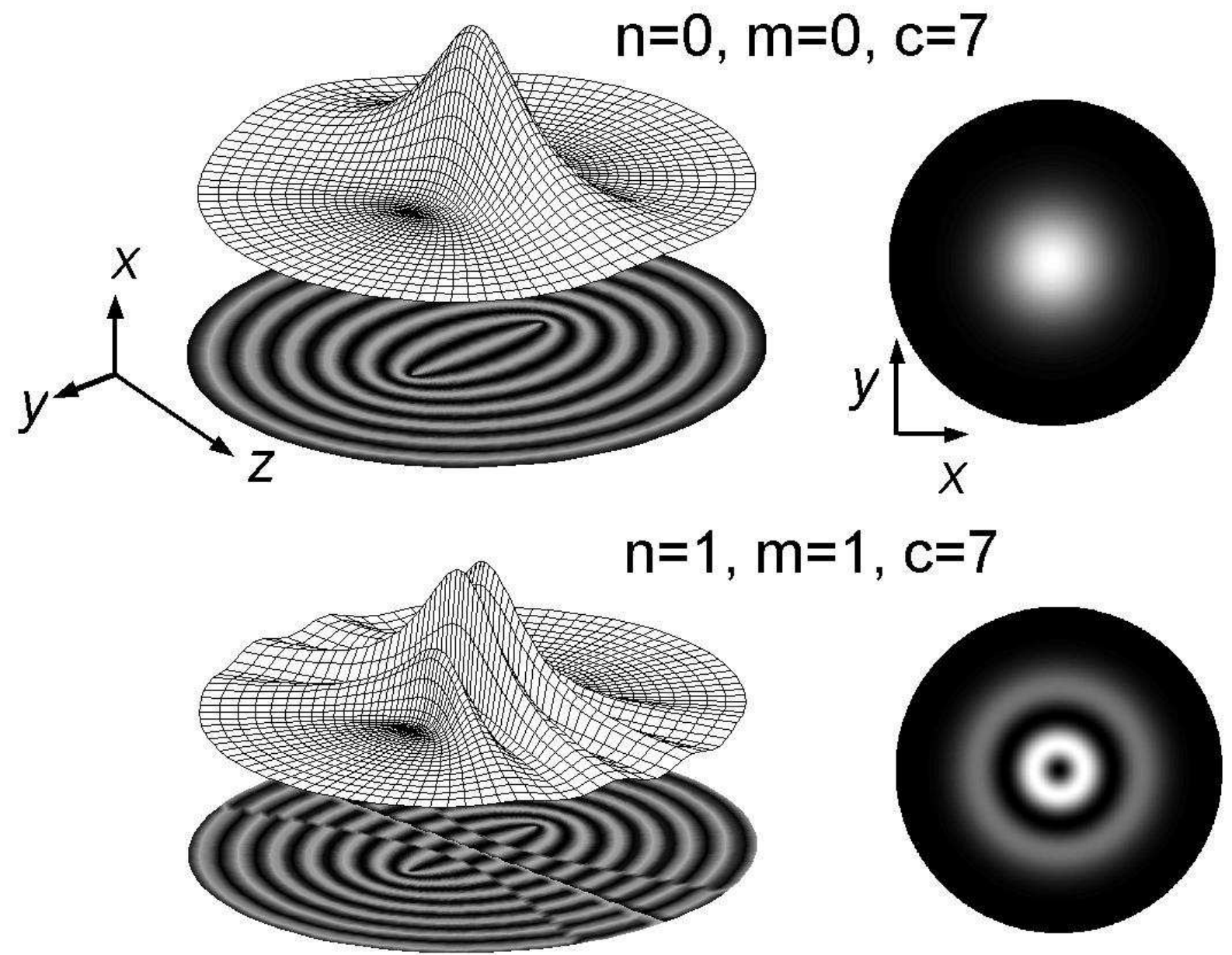


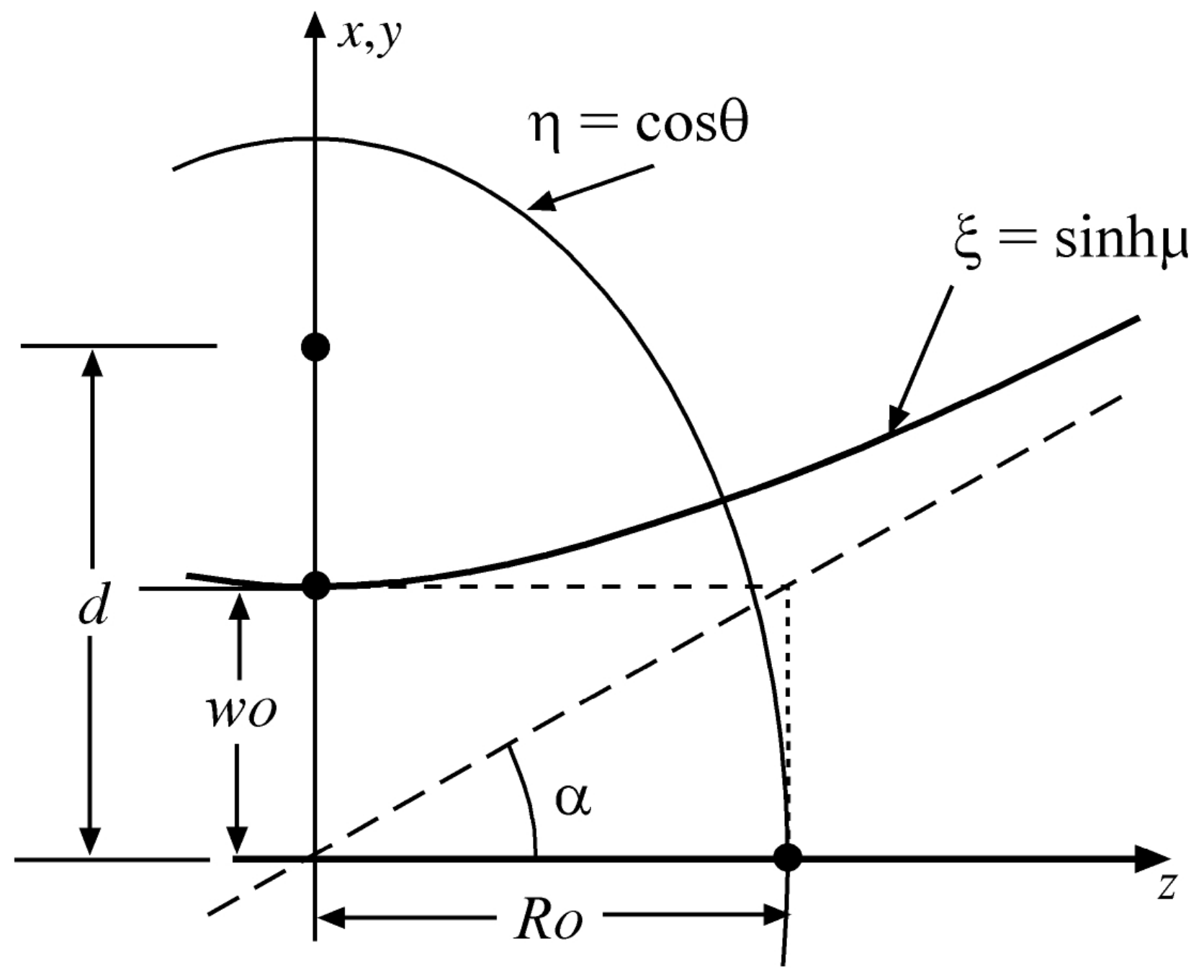



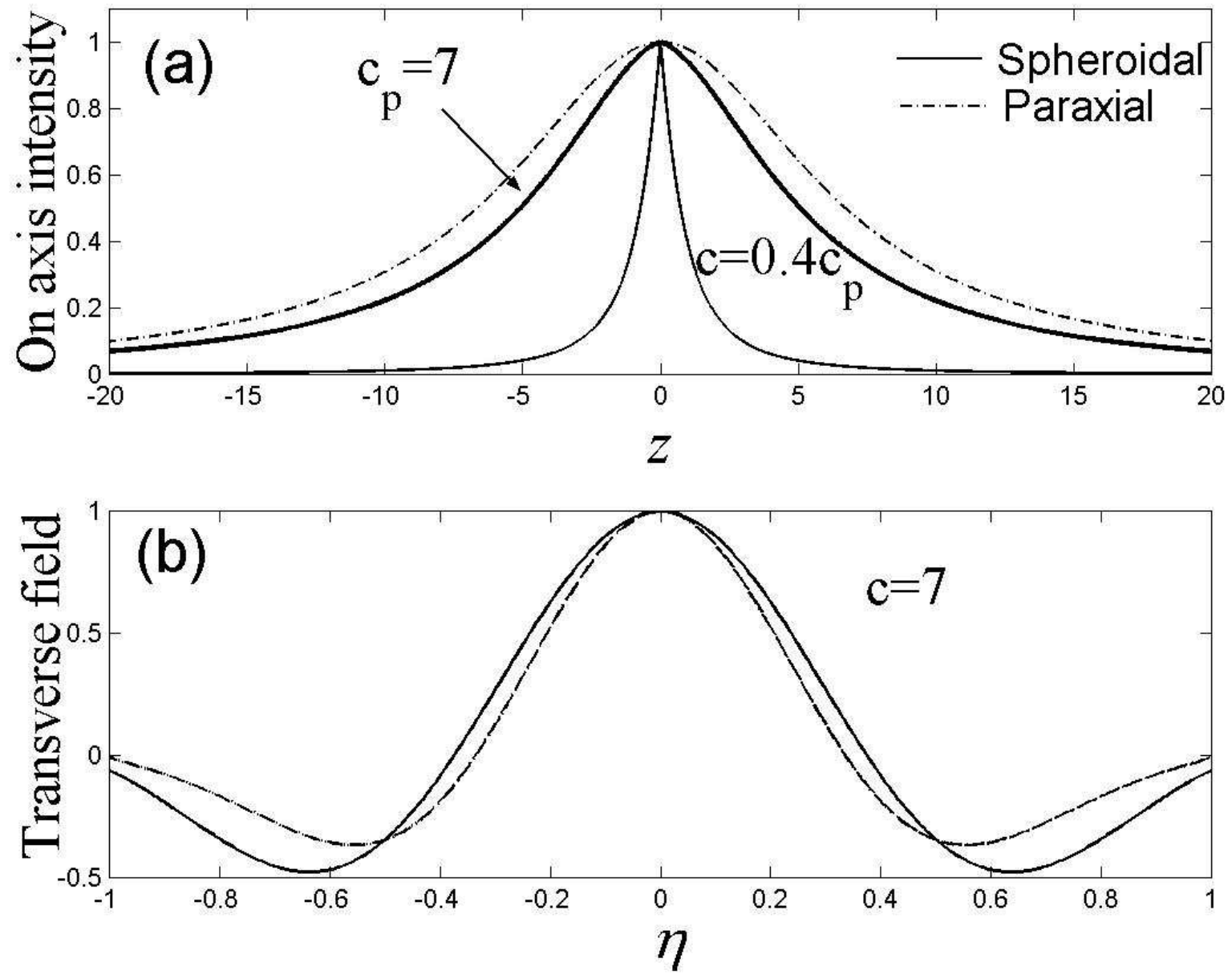